\title{
Non-adherence among women enrolled in a contraceptive vaginal ring use study in Kisumu, Kenya, 2014-2015
}

\author{
Eleanor McLellan-Lemal', Roman Gvetadze', Mitesh A Desai ${ }^{1}$, Esther M Makanga², Yi Pan', Richard E Haaland', \\ Angela N Holder ${ }^{1}$, Victor Mudhune ${ }^{2}$, Tiffany Williams' ${ }^{1}$, Taraz Samandari ${ }^{1}$ \\ ${ }^{1}$ Centers for Disease Control and Prevention, Office of Infectious Diseases, National Center for HIV/AIDS, Viral Hepatitis, STD, and TB Prevention, \\ Division of HIVIAIDS Prevention, Atlanta, Georgia, USA, ${ }^{2}$ Kenya Medical Research Institute, Center for Global Health Research, Kisumu, Kenya \\ Keywords: global health \\ https://doi.org/10.29392/joghr.2.e2018032
}

\section{Journal of Global Health Reports}

Vol. 2, 2018

\section{Background}

Given future potential use of vaginal rings to prevent HIV infection, we examined the association of contraceptive vaginal ring (CVR) non-adherence with user dissatisfaction, tolerability, demographic, and behavioral factors.

\section{Methods}

In an open-label single-group study, sexually active women aged 18-34 years using oral or injectable hormonal contraception, conveniently sampled from general population, were assigned to 6-month use of a commercial CVR currently not licensed for use in Kenya. Non-adherence in any CVR cycle completed was assessed from: (1) self-report (not used for at least 1 day), and (2) pharmacy record (failure to timely receive a new CVR or return a used one). Additionally, non-adherence was assessed in a subset of participants by residual progestin and estrogen levels measured in returned CVRs.

\section{Results}

Of 202 participants who underwent CVR insertion by a study clinician, 142 completed all 6 visits, 172 responded to questions about ring use, and 43 provided used CVRs from months 1,3 , and 6 for residual hormone analysis. Non-adherence was $14.0 \%(24 / 172)$ by self-report and $54.5 \%(110 / 202)$ by pharmacy record. Non-adherence by pharmacy record was significantly reduced among women with a salary-based income (prevalence ratio (PR) $0.71,95 \%$ confidence interval (CI) (0.55-0.91)] compared to women with income not salary-based or no income. Participants dissatisfied with CVR on $\geqslant 4$ aspects (ambiguity of instructions, inconvenience of use, sensation, sexual discomfort, etc.) were more likely to report non-adherence (PR 2.69, 95\% $\mathrm{CI}=(1.31-5.52)]$ compared to those dissatisfied with $\leqslant 3$ aspects. Non-adherence by residual hormone levels was identified in $46.5 \%$ (20/43) participants. Over time, this subset of participants showed increasing non-adherence $(P=0.004)$. We found lack of agreement among the various measures of non-adherence.

\section{Conclusions}

Economic empowerment interventions, especially those emphasizing partner-independent income options, and expanded education on CVR features may alleviate non-adherence. Addressing CVR dissatisfaction preemptively may also help mitigate non-adherence.

Vaginal rings that can deliver an antiretroviral (ARV) drug for preventing HIV infection, separately or in combination with agents for preventing pregnancy or sexually transmitted infections (STIs), are under development. ${ }^{1-3}$ HIV prevention researchers anticipate that vaginal delivery of ARV drugs by long-acting (i.e., >28-day) methods will help avoid various adherence issues observed for some women with oral daily ${ }^{4}$ or peri-coitally dosed pre-exposure prophylaxis (PrEP). ${ }^{5}$ Contraceptive vaginal rings (CVRs) may offer valuable insights on users' experiences, including potential adherence challenges associated with use of this technology, in particular among first-time users.

Worldwide, an overall low estimated prevalence $(0.1 \%)$ of vaginal contraceptive methods, including CVRs, among women 15 to 49 years of age ${ }^{6}$ raises concerns about potential uptake (i.e., action taken to initiate use) and use of a multipurpose ring. Explanations for low uptake of CVRs include challenges with vaginal insertion, foreign-body sensation, ${ }^{7}$ sexual interference, ${ }^{8}$ and concerns about the ring getting lost inside the body. ${ }^{9}$ Poor CVR promotion, cost, and 
short duration of use (e.g., 21-day use cycle) further account for CVR uptake obstacles. ${ }^{10}$ While high satisfaction, tolerability, favorable bleeding control, and adherence have been reported by women initiating CVR use, ${ }^{3}$ a continuation rate of $26 \%$ after 6 months by new CVR users has been observed, which is even lower than the $29 \%$ reported for combined oral contraceptives. ${ }^{11}$

Adherence in clinical trials is subject to overestimation given challenges with measuring product use, both behaviorally and biologically. ${ }^{12}$ Nonetheless, adherence in a trial is expected to be as good as or greater than "real world" use, where social, structural, and behavioral interventions may need to be coupled with biomedical ones and directed at providers and target populations alike. ${ }^{13}$ Women's empowerment, defined as the "ability to make effective choices and to transform these choices into desired outcomes" as opposed to merely following prescriptive conventions, ${ }^{14}$ is a critical component in acceptability, satisfaction, and adherence of female-focused health interventions, including contraception. Women's empowerment, which is influenced by individual, relational, and social determinants of health (e.g., socioeconomic status, education, gender dynamics, healthcare access, availability of methods, etc.), intersects with perceptions related to product properties, including the safety profile.

Given the seemingly apparent reasons that women would be interested in CVR use, it stands to reason that to optimize adherence, we need a better understanding of women's experiences with this technology, in particular barriers to use. To address this issue, we examined the association of CVR non-adherence with user dissatisfaction, tolerability, demographic, and behavior variables among firsttime users of this technology in Kisumu, Kenya. Of note, no CVR is licensed currently for use in Kenya, and the target population in this setting was predominantly naïve to CVRs. Our analysis sample included women who had at least one follow-up monthly visit post-CVR insertion. Two additional sub-analyses were performed for participants who provided follow-up behavioral data or were selected purposively for assessing residual progestin and estrogen levels in returned CVRs.

\section{MATERIALS AND METHODS}

Between April 2014 and August 2015, an open-label singlegroup study of NuvaRing ${ }^{\circledR}$ (Sharp \& Dohme B.V., a subsidiary of Merck \& Co., Inc, Kenilworth, NJ, USA) use was conducted in Kisumu, Kenya. In brief, a convenience sample of women was recruited from family planning and reproductive health clinics within Kisumu County via help of 10 community health volunteers and participant word-ofmouth referrals without incentives. We enrolled women who were 18-34 years of age, resided within a 150-kilometer radius of Kisumu City with no plans for relocation in the next 12 months, were fluent in English, Swahili, or Dholuo, provided documentation of depot medroxyprogesterone acetate (DMPA) or oral contraceptive pills (OCPs) use in the past three months, self-reported $\geqslant 2$ episodes of vaginal intercourse on different days in the past 30 days at screening, tested negative for pregnancy and HIV, had no current or history of known medical contraindications for CVR use, were not breastfeeding or within three months of parturition at screening, and were prepared to use the CVR for six months in place of injectable or oral contraceptives. Condom use for HIV and STI prevention was strongly encouraged.

Written informed consent was obtained prior to data and sample collection. Ring initiation schedules varied depending on completion of last OCP or DMPA-use cycle. OCP users were able to initiate ring use as soon as 30 days following study enrollment, while DMPA users may have had to delay ring initiation up to three months. To avoid overlap use in contraceptives, discontinuation of DMPA and initiation of NuvaRing ${ }^{\circledR}$ were recommended on the due date for the next injection. The informed consent process and data collection were available in the language choice of the participant (English, Swahili, or Dholuo). After receiving instruction and demonstration on a 3-dimensional female pelvic model, ring self-insertion and removal practice training occurred at the study clinic office for 210 women. At each follow-up visit, women received a bar of soap and 500 Kenya Shillings (approximately US\$ 5). In addition, as a part of the study clinic's standard services, women received feminine sanitary pads, condoms, and hormonal contraceptives (at study exit or CVR discontinuation). Participants and their children were eligible to present to the study clinic at any time for diagnosis of common ailments and, if appropriate, referral for treatment. Participants' sexual partners were entitled to receive free STI treatment following syndromic management assessment.

\section{DATA COLLECTION}

Non-adherence assessment was performed at follow-up visits scheduled between day 21 and day 29 of each one-month CVR cycle for six months following CVR initiation. At each visit, an electronic pharmacy log was used to record dates of each CVR dispensation and return of used CVRs by each participant. Of note, CVR dispensation and follow-up CVR visits occurred on the same date.

Demographic as well as baseline and quarterly behavioral data were collected using audio computer-assisted self-interview (ACASI). CVR user experiences and non-adherence were assessed using an adapted version of the $\mathrm{Nu}$ vaRing ${ }^{\circledR}$ questionnaire developed by Novak et al, ${ }^{15}$ administered via computer-assisted personal interview (CAPI). Our questionnaire covered five broad CVR dimensions: difficulty of use, ambiguity of instructions, sexual discomfort, non-compliance, dissatisfaction. Willingness to recommend the CVR to others was also assessed. Within the dissatisfaction dimension, questions centered on specific CVR aspects (e.g., insertion, removal, placement, package use, physical comfort, partner support).

Testing for pregnancy was undertaken at each monthly visit. Pregnant women discontinued CVR use, received local antenatal care clinic referrals, and participated in quarterly follow-up. Rapid HIV testing was performed at baseline and every three months thereafter. Testing for other STIs and bacterial vaginosis was completed at baseline and study exit. Vaginal swabs were used to collect samples for gonorrhea, chlamydia, and bacterial vaginosis testing. Blood 
samples were collected for herpes simplex virus type 2 (HSV-2) and syphilis testing.

\section{MEASURES}

Our primary outcomes were specified as binary variables summarizing different types of CVR non-adherence based on: (a) self-report, (b) pharmacy record, and (c) residual hormone levels. Participants were classified as non-adherent by self-report if they reported during follow-up any missed CVR use. Non-adherence by pharmacy record was determined if a new ring was not dispensed between days 19-31 for any CVR cycle or a used ring was not returned for every CVR cycle completed.

An objective measure of non-adherence was assessed by analyzing residual etonogestrel (progestin) and ethinyl estradiol (estrogen) levels in returned CVRs for months 1 $(n=26), 3(n=43)$, and $6(n=43)$ for a subset of participants purposively selected from those who self-reported perfect (100\%) adherence at all monthly visits (henceforward referred to as the hormone analysis sub-sample). NuvaRing ${ }^{\circledR}$ contains 11.7 milligrams $(\mathrm{mg})$ of progestin, and $2.7 \mathrm{mg}$ of estrogen. The average release rates per 24 hours over the 3-week use cycle for progestin and estrogen are $0.120 \mathrm{mg}$ and $0.015 \mathrm{mg}$, respectively. ${ }^{16}$ Participants were classified as non-adherent if returned CVRs showed residual progestin or estrogen levels greater than $95 \%$ of those measured in a new, never used ring. Residual hormone levels were defined as consistent if the same use indicators were present across all returned CVRs examined (i.e., 100\% overall use or 100\% overall non-use).

Responses to demographic questions were coded as categorical variables. Incident STI or bacterial vaginosis, and responses to most risk behavior questions were included as binary variables. CVR dissatisfaction was characterized by a binary variable indicating displeasure with more than three CVR aspects within the four negative attitude domains, which included inconvenience of ring use (including package use), ambiguity of instructions, sexual discomfort, and difficulty with compliance. The number of dissatisfaction aspects reported at each CVR follow-up visit was used to visually depict dissatisfaction over time. Lastly, willingness to recommend the CVR to others was based a 5-point Likert agreement scale (highly agree, agree, undecided, disagree, and highly disagree).

Tolerability was summarized as a binary variable that distinguished between reports of $\geqslant 2$ side effects (SEs) during the CVR-use period and reports of 1 or no SE. SEs included elevated systolic $(>160 \mathrm{mmHg}$ ) or diastolic $(>110$ $\mathrm{mmHg}$ ) blood pressure, self-reported fatigue, vaginal discharge, genital pain, headaches, depression, and abnormal vaginal bleeding. The number of SEs reported at each follow-up visit was also used to graphically depict tolerability over time.

\section{STATISTICAL METHODS}

We examined the factors associated with non-adherence among women who initiated ring use and completed at least one follow-up visit (non-adherence sample), in the subsample of participants who provided responses on ACASI questionnaire (behavioral sub-sample), and in the sub-sample of participants included in the residual hormone-level analysis. Descriptive statistics were used to summarize categorical (frequency and percentage) and continuous (mean, median, standard deviation, and range) variables. The association with non-adherence outcomes was summarized by prevalence ratio (PR) and robust 95\% confidence interval (CI) estimated from a log-binomial regression model using the generalized estimating equations (GEE) approach. ${ }^{17,18}$ The same GEE log-binomial regression with the visit number treated as a continuous covariate was used to model the over-time trend in a binary characteristic (dissatisfaction and tolerability). The incidence rate for pregnancy or HIV infection was estimated as the number of respective events occurred over study follow-up per 100 person-years with a robust 95\% CI=obtained from a GEE Poisson model. Agreement among non-adherence measures was assessed by Cohen's kappa. ${ }^{19}$ All statistical tests were two-sided and interpreted at 0.05 level of significance. The analyses were performed in SAS 9.3 (SAS Institute Inc., Cary, NC, USA).

\section{RESULTS}

From the women pre-screened, 29.2\% (202/692) initiated CVR use and completed at least one follow-up visit (Figure 1). Overall, three HIV seroconversions occurred during the study for an incidence rate of 3.6 per 100 person-years (95\% $\mathrm{CI}=1.2-11.0$ ) (data not shown). Among the non-adherence sample, 91.6\% (185/202) who provided responses to ACASI questions were included in the behavioral sub-sample.

The non-adherence sample $(n=202)$ accrued 83.7 out of the expected total 101 (82.9\%) person-years of observation. Participants were followed for a median of 5.3 months, with follow-up ranging from 0.7 to 6.5 months (mean=5.0 months, standard deviation=1.1 months). Demographic characteristics of our CVR non-adherence sample are presented in Table 1. Slightly over three-fourths of participants (79.7\%) were using DMPA prior to initiating ring use.

Out of the 202 participants, $85.1 \%$ (172) self-reported on CVR non-adherence. Overall, $14.0 \%$ (24/172) and 54.5\% $(110 / 202)$ were non-adherent by self-report and by pharmacy record, respectively (Table 1 ). Over a total of 83.7 person-years of follow-up, five pregnancies occurred yielding a pregnancy rate of 6.0 per 100 person-years $(95 \%$ $\mathrm{CI}=2.5-14.3$ ) (data not shown).

The only significant factor associated with non-adherence by pharmacy record was the main source of personal income (Table 1). Specifically, the prevalence of non-adherence by pharmacy record was reduced by $29 \%$ among women with salary-based income compared to those with no personal income or no income $(\mathrm{PR}=0.71,95 \%$ $\mathrm{CI}=0.55-0.91, P=0.008]$. CVR dissatisfaction was the only significant factor associated with non-adherence by self-report. Women indicating dissatisfaction with $>3$ CVR-related aspects (e.g., ring properties, use features) were more likely to be non-adherent by self-report compared to women dissatisfied with $\leqslant 3$ aspects $(\mathrm{PR}=2.69 \mathrm{CI}=1.31-5.52, P=0.007)$.

Figure 2 depicts the proportion of participants $(n=202)$ reporting any CVR dissatisfaction by study follow-up visit. Out of 491 dissatisfaction aspects reported across all follow-up visits, sexual discomfort accounted for $33.6 \%$ of dis- 
Table 1. Self-reported and pharmacy record non-adherence by demographic, satisfaction, and tolerability factors ( $\mathrm{n}=202)$, Kisumu contraceptive vaginal ring study, Kisumu, Kenya, 2014-2015"

\begin{tabular}{|c|c|c|c|c|c|c|c|c|c|c|}
\hline \multirow[b]{2}{*}{ Predictor } & \multicolumn{4}{|c|}{$\begin{array}{c}\text { Non-adherence by self-report } \dagger \\
n=24 / 172(14.0 \%)\end{array}$} & \multirow[b]{2}{*}{$P$-value } & \multicolumn{5}{|c|}{$\begin{array}{l}\text { Non-adherence by pharmacy record } \neq \\
n=110 / 202(54.5 \%)\end{array}$} \\
\hline & $\begin{array}{l}\text { Total, } n \\
\text { (col\%) }\end{array}$ & $\begin{array}{l}\text { Non- } \\
\text { adherent, n } \\
\text { (col \%) }\end{array}$ & $\begin{array}{l}\text { Prevalence } \\
\text { (\%) }\end{array}$ & $\begin{array}{l}\text { Prevalence ratio } \\
\quad(95 \% \mathrm{Cl})\end{array}$ & & $\begin{array}{l}\text { Total, } n \\
\text { (col\%) }\end{array}$ & $\begin{array}{c}\text { Non- } \\
\text { adherent, } \\
\text { n (col \%) }\end{array}$ & $\begin{array}{l}\text { Prevalence } \\
\text { (\%) }\end{array}$ & $\begin{array}{l}\text { Prevalence ratio } \\
\quad(95 \% \mathrm{Cl})\end{array}$ & $P$-value \\
\hline Age at screening (years): & & & & & 0.542 & & & & & 0.150 \\
\hline $18-24$ & $90(52.3)$ & $15(62.5)$ & 16.7 & $1.28(0.40,4.04)$ & 0.677 & $104(51.5)$ & $52(47.3)$ & 50.0 & $1.04(0.67,1.61)$ & 0.865 \\
\hline $25-29$ & 59 (34.3) & $6(25.0)$ & 10.2 & $0.78(0.21,2.86)$ & 0.707 & $71(35.1)$ & 45 (40.9) & 63.4 & $1.32(0.86,2.02)$ & 0.210 \\
\hline $30-34$ & $23(13.4)$ & $3(12.5)$ & 13.0 & Ref. & & $27(13.4)$ & $13(11.8)$ & 48.1 & Ref. & \\
\hline Marital status: & & & & & 0.459 & & & & & 0.559 \\
\hline Single & $30(17.9)$ & $5(21.7)$ & 16.7 & $0.72(0.20,2.58)$ & 0.617 & $31(15.7)$ & $19(17.3)$ & 61.3 & $1.43(0.73,2.78)$ & 0.293 \\
\hline Married/cohabiting & $125(74.4)$ & $15(65.2)$ & 12.0 & $0.52(0.17,1.56)$ & 0.244 & $153(77.3)$ & $85(77.3)$ & 55.6 & $1.30(0.70,2.41)$ & 0.413 \\
\hline Other & $13(7.7)$ & $3(13.0)$ & 23.1 & Ref. & & $14(7.1)$ & $6(5.5)$ & 42.9 & Ref. & \\
\hline Religion: & & & & & 0.119 & & & & & 0.549 \\
\hline Roman Catholic & $76(44.4)$ & $11(47.8)$ & 14.5 & $0.58(0.24,1.40)$ & 0.225 & $89(44.3)$ & $51(46.4)$ & 57.3 & $0.97(0.69,1.35)$ & 0.837 \\
\hline Other Christian & $71(41.5)$ & $6(26.1)$ & 8.5 & $0.34(0.12,0.95)$ & 0.040 & $80(39.8)$ & $40(36.4)$ & 50.0 & $0.84(0.59,1.21)$ & 0.351 \\
\hline Other non-Christian & $24(14.0)$ & $6(26.1)$ & 25.0 & Ref. & & $32(15.9)$ & $19(17.3)$ & 59.4 & Ref. & \\
\hline \multicolumn{11}{|c|}{ Highest education completed: } \\
\hline Primary or less & $116(68.2)$ & $15(65.2)$ & 12.9 & $0.87(0.39,1.93)$ & 0.737 & $135(67.5)$ & $76(69.7)$ & 56.3 & $1.11(0.84,1.47)$ & 0.472 \\
\hline Secondary or higher & $54(31.8)$ & $8(34.8)$ & 14.8 & Ref. & & $65(32.5)$ & $33(30.3)$ & 50.8 & Ref. & \\
\hline \multicolumn{11}{|l|}{ Employment status: } \\
\hline Employed & $107(62.9)$ & $11(47.8)$ & 10.3 & $0.54(0.25,1.15)$ & 0.110 & $126(63.0)$ & $66(60.6)$ & 52.4 & $0.90(0.70,1.16)$ & 0.426 \\
\hline Unemployed & $63(37.1)$ & $12(52.2)$ & 19.0 & Ref. & & $74(37.0)$ & $43(39.4)$ & 58.1 & Ref. & \\
\hline \multicolumn{11}{|c|}{ Main source of personal income: } \\
\hline Salary-based & $88(51.5)$ & $9(39.1)$ & 10.2 & $0.61(0.28,1.33)$ & 0.210 & $105(52.2)$ & $48(43.6)$ & 45.7 & $0.71(0.55,0.91)$ & 0.008 \\
\hline $\begin{array}{l}\text { Not salary-based/ } \\
\text { None }\end{array}$ & $83(48.5)$ & $14(60.9)$ & 16.9 & Ref. & & $96(47.8)$ & $62(56.4)$ & 64.6 & Ref. & \\
\hline Parity: & & & & N/A & N/A & & & & & 0.407 \\
\hline 0 live births & $10(5.9)$ & $0(0.0)$ & 0.0 & & & $14(7.0)$ & $8(7.3)$ & 57.1 & $0.95(0.58,1.54)$ & 0.831 \\
\hline 1-2 live births & $88(51.8)$ & $15(65.2)$ & 17.0 & & & $103(51.5)$ & $52(47.3)$ & 50.5 & $0.84(0.65,1.09)$ & 0.181 \\
\hline 3 or more live births & $72(42.4)$ & $8(34.8)$ & 11.1 & & & $83(41.5)$ & $50(45.5)$ & 60.2 & Ref. & \\
\hline
\end{tabular}




\begin{tabular}{|c|c|c|c|c|c|c|c|c|c|c|}
\hline & \multicolumn{5}{|c|}{$\begin{array}{c}\text { Non-adherence by self-report } \dagger \\
n=24 / 172(14.0 \%)\end{array}$} & \multicolumn{5}{|c|}{$\begin{array}{c}\text { Non-adherence by pharmacy record } \neq \\
n=110 / 202(54.5 \%)\end{array}$} \\
\hline DMPA & $135(78.5)$ & $16(66.7)$ & 11.9 & $0.55(0.25,1.18)$ & 0.124 & $161(79.7)$ & $87(79.1)$ & 54.0 & $0.96(0.71,1.31)$ & 0.811 \\
\hline OCPs & $37(21.5)$ & $8(33.3)$ & 21.6 & Ref. & & $41(20.3)$ & $23(20.9)$ & 56.1 & Ref. & \\
\hline \multicolumn{11}{|l|}{ Age (years) of first sex: } \\
\hline 14 or less & $35(25.5)$ & $7(35.0)$ & 20.0 & $1.57(0.68,3.62)$ & 0.290 & $45(27.8)$ & $28(32.6)$ & 62.2 & $1.26(0.94,1.68)$ & 0.127 \\
\hline 15 or older & $102(74.5)$ & $13(65.0)$ & 12.7 & Ref. & & $117(72.2)$ & $58(67.4)$ & 49.6 & Ref. & \\
\hline \multicolumn{11}{|c|}{ History of unwanted/forced sex: } \\
\hline Yes & $57(33.3)$ & $9(39.1)$ & 15.8 & $1.29(0.59,2.79)$ & 0.525 & $64(31.8)$ & $38(34.5)$ & 59.4 & $1.13(0.87,1.46)$ & 0.353 \\
\hline No & $114(66.7)$ & $14(60.9)$ & 12.3 & Ref. & & $137(68.2)$ & $72(65.5)$ & 52.6 & Ref. & \\
\hline \multicolumn{11}{|c|}{ Pregnancy intentions in next 12 months: } \\
\hline $\begin{array}{l}\text { Intends/desires to get } \\
\text { pregnant }\end{array}$ & $8(4.8)$ & $1(5.0)$ & 12.5 & $1.05(0.16,6.91)$ & 0.957 & $8(4.7)$ & $4(5.2)$ & 50.0 & $1.10(0.54,2.25)$ & 0.788 \\
\hline $\begin{array}{l}\text { No intent/desire to get } \\
\text { pregnant }\end{array}$ & $160(95.2)$ & $19(95.0)$ & 11.9 & Ref. & & $161(95.3)$ & $73(94.8)$ & 45.3 & Ref. & \\
\hline \multicolumn{11}{|l|}{ Incident pregnancy: } \\
\hline Yes & $5(2.9)$ & $2(8.3)$ & 40.0 & $3.04(0.97,9.51)$ & 0.057 & $5(2.5)$ & $2(1.8)$ & 40.0 & $0.73(0.25,2.15)$ & 0.568 \\
\hline No & $167(97.1)$ & $22(91.7)$ & 13.2 & Ref. & & $197(97.5)$ & $108(98.2)$ & 54.8 & Ref. & \\
\hline \multicolumn{11}{|c|}{ Would recommend CVR to others: } \\
\hline Agrees & $154(90.6)$ & $23(95.8)$ & 14.9 & $2.39(0.35,16.54)$ & 0.378 & $182(91.9)$ & $101(93.5)$ & 55.5 & $1.27(0.72,2.24)$ & 0.414 \\
\hline Strongly agrees & $16(9.4)$ & $1(4.2)$ & 6.3 & Ref. & & $16(8.1)$ & $7(6.5)$ & 43.8 & Ref. & \\
\hline \multicolumn{11}{|l|}{ CVR dissatisfaction:§ } \\
\hline $\begin{array}{l}\text { More than } 3 \\
\text { dissatisfaction aspects }\end{array}$ & $43(30.5)$ & $13(54.2)$ & 30.2 & $2.69(1.31,5.52)$ & 0.007 & $47(29.0)$ & $27(30.0)$ & 57.4 & $1.05(0.78,1.41)$ & 0.754 \\
\hline $\begin{array}{l}3 \text { or less dissatisfaction } \\
\text { aspects }\end{array}$ & $98(69.5)$ & $11(45.8)$ & 11.2 & Ref. & & $115(71.0)$ & $63(70.0)$ & 54.8 & Ref. & \\
\hline \multicolumn{11}{|l|}{ CVR tolerability: } \\
\hline $\begin{array}{l}\text { Side effect (SE) } \\
\text { reported more than } \\
\text { once }\end{array}$ & $28(16.8)$ & $4(18.2)$ & 14.3 & $1.10(0.40,3.01)$ & 0.848 & $34(18.8)$ & $18(19.8)$ & 52.9 & $1.07(0.75,1.52)$ & 0.725 \\
\hline 0 to $1 \mathrm{SE}$ reported & $139(83.2)$ & $18(81.8)$ & 12.9 & Ref. & & $147(81.2)$ & $73(80.2)$ & 49.7 & Ref. & \\
\hline
\end{tabular}




\begin{tabular}{|c|c|c|c|c|c|c|c|c|c|c|}
\hline \multirow[b]{2}{*}{ Negative } & \multicolumn{5}{|c|}{$\begin{array}{l}\text { Non-adherence by self-report } \dagger \\
\quad n=24 / 172(14.0 \%)\end{array}$} & \multicolumn{5}{|c|}{$\begin{array}{l}\text { Non-adherence by pharmacy record } \neq \\
n=110 / 202(54.5 \%)\end{array}$} \\
\hline & $26(21.7)$ & $4(25.0)$ & 15.4 & $1.21(0.42,3.43)$ & 0.726 & $29(20.4)$ & $15(19.2)$ & 51.7 & $0.93(0.63,1.37)$ & 0.705 \\
\hline Neutral or positive & $94(78.3)$ & $12(75.0)$ & 12.8 & Ref. & & $113(79.6)$ & $63(80.8)$ & 55.8 & Ref. & \\
\hline
\end{tabular}

$\mathrm{CI}$ - confidence interval, DMPA - depot medroxyprogesterone acetate, OCPs - oral contraceptive pills, CVR - contraceptive vaginal ring

"Sample sizes fluctuate slightly for some variables due to missing data. Some percentages do not sum to 100 because of rounding.

$\dagger$ Due to incomplete or missing data, self-reported data was available for 85.1\% (172/202) of the $202 \mathrm{CVR}$ non-adherence sample.

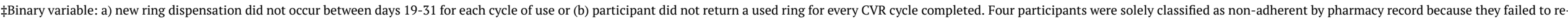
turn a used CVR for at least one follow-up visit.

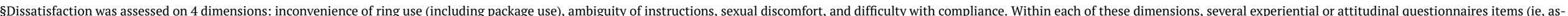

pects) were addressed. 


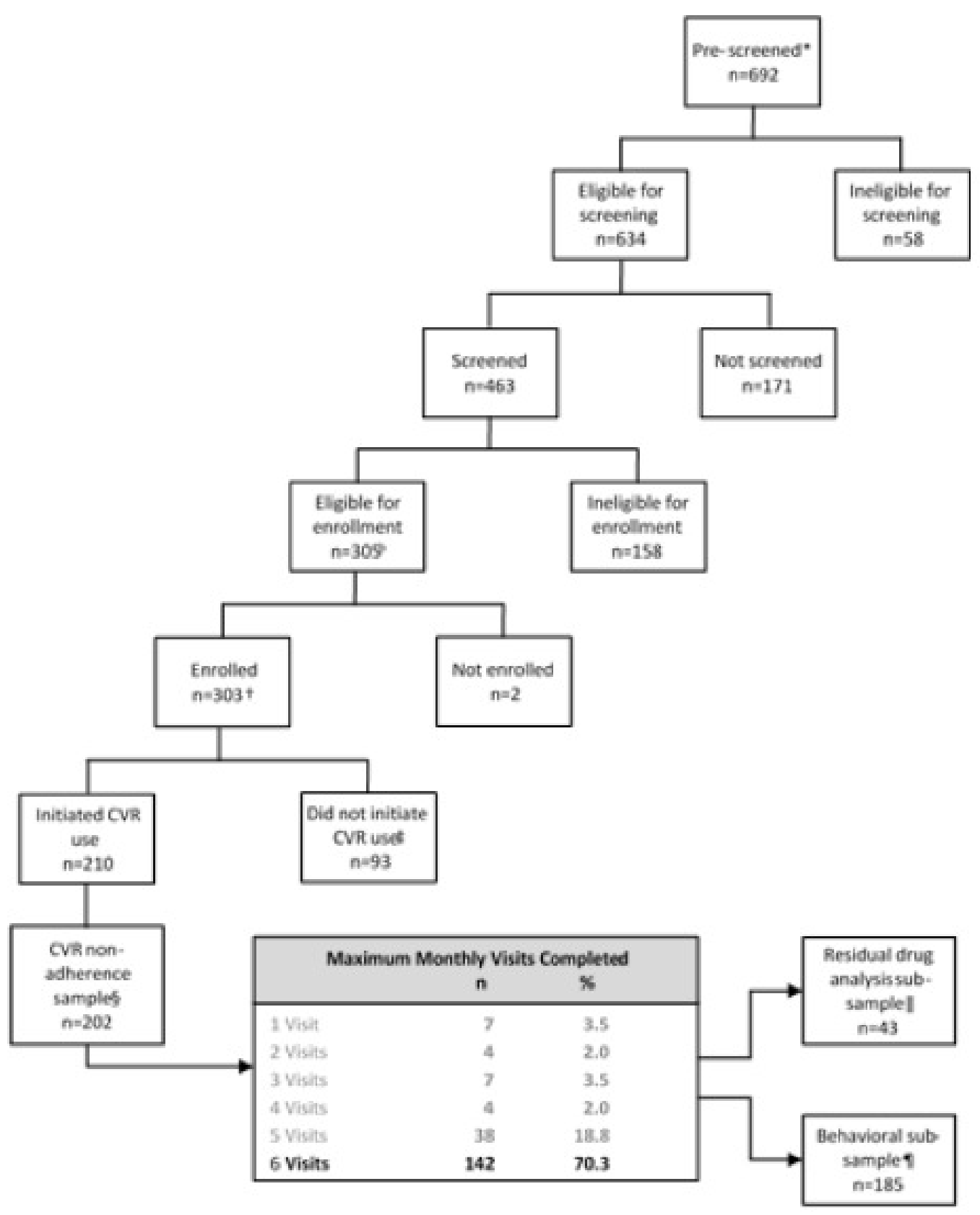

Figure 1. Flowchart: Screening, enrollment, and follow-up, Kisumu contraceptive vaginal ring study, Kisumu, Kenya, 2014-2015.

*Information on participants who went from pre-screening to enrollment has been published elsewhere. ${ }^{15} \dagger$ An additional participant was identified after study close out and preparation of final dataset. The number eligible for enrollment and the number enrolled differ by one from previously reported data, ${ }^{15} 304$ and 302 , respectively. $\ddagger$ Among the 93 enrollees who did not initiate contraceptive vaginal ring (CVR) use, $43.0 \%$ withdrew their study participation, $45.2 \%$ were lost to follow-up, and $12.0 \%$ were no longer medically eligible. §Women initiating CVR use who completed at least one adherence follow-up visit; $8 / 210$ (3.8\%) did not have any follow-up time: 5 withdrew participation, 2 were medically discontinued, and 1 was lost to follow-up. ||Overall, 112 returned CVRs were assessed among 43 participants who self-reported perfect (100\%) adherence at all monthly visits. At month 1,26 returned CVRs were used to measure residual drug levels and to assess non-adherence. ${ }^{18}$ Forty-three CVRs were assessed at months 3 and $6.9 \mathrm{CVR}$ users who completed one or more ACASI questionnaires in which $\geqslant 1$ month CVR experience occurred.

satisfactions (some participants may have reported this aspect at multiple visits).

SEs were reported by $9-14 \%$ of participants each month (Figure 3). The three most commonly reported SEs were headache, fatigue, and vaginal discharge. We found no significant associations between CVR tolerability and non-adherence either by self-report or by pharmacy record. All reported SEs were mild in severity (Grade 1) and did not require treatment.

Table 2 displays non-adherence by self-report and by pharmacy record in the behavioral sub-sample $(n=185)$. Out of the 185 participants, 84.3\% (156) self-reported on CVR non-adherence. Relevant ACASI data were available for 19 of 24 participants who were non-adherent by self-report and $96.4 \%(106 / 110)$ of participants who were non-adherent 


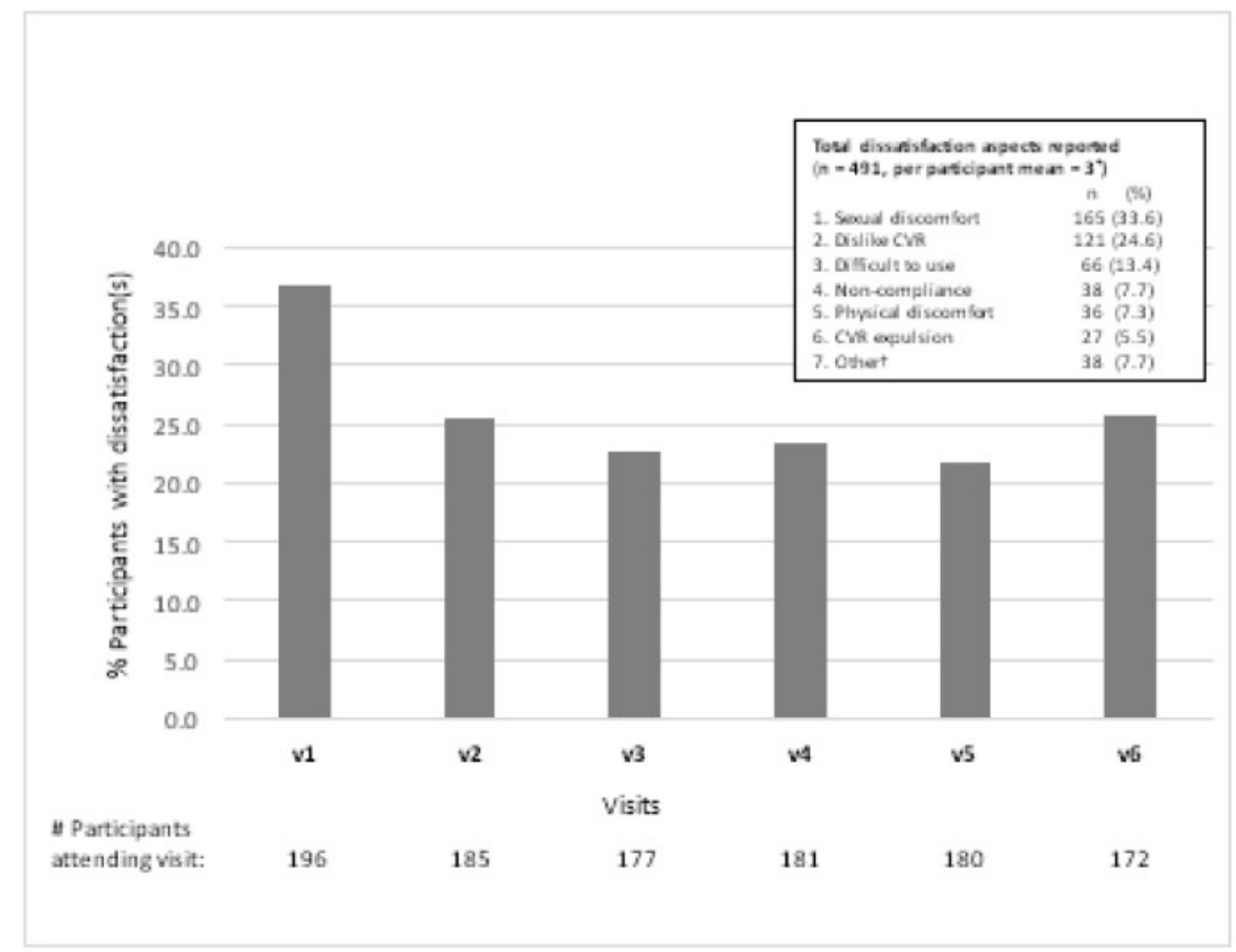

Figure 2. Proportion of participants $(n=202)$ reporting $\geqslant 1$ dissatisfaction aspect by Kisumu contraceptive vaginal ring study visit, Kisumu, Kenya, 2014-2015.

*Cumulative count of dissatisfaction aspects reported by all participants over study follow-up; the per participant mean represents the average cumulative count of dissatisfaction aspects reported over follow-up. †Other category includes lack of partner support $(\mathrm{n}=8)$, unclear instructions $(\mathrm{n}=5)$, and miscellaneous responses ( $\mathrm{n}=25)$.

by pharmacy record. We found no evidence of an association between the behavioral measures and non-adherence by self-report or by pharmacy record (Table 2).

In the analysis of residual progestin and estrogen, a total of 112 returned CVRs were examined for 43 participants who self-reported perfect adherence at all monthly visits. Overall, 20 (46.5\%) out of 43 participants were non-adherent by residual hormone levels, including 7 (16.3\%) who showed consistent non-use and 13 (30.2\%) who showed partial or inconclusive use (consistent with tampering). Analysis of residual hormone levels in returned CVRs showed increasing non-adherence over time $(P=0.004$, nonadherence for months 1,3 , and 6 were $19.2 \%, 32.6 \%$, and $44.2 \%$, respectively).

Finally, we observed poor to no agreement (Cohen's kappa $=0.03$ ) between pharmacy record and self-reported non-adherence measures. There was disagreement observed between pharmacy record non-adherence and residual drug analysis as well as between self-reported non-adherence and residual drug analysis (data not shown).

\section{DISCUSSION}

We sought to understand the drivers of non-adherence to a CVR in a naïve population, with an intent to incorporate lessons learned into future studies of multipurpose HIV prevention rings in western Kenya. Despite all first-time users of CVR in our study being willing to recommend the ring to other women, which suggests that this technology for at least contraceptive purposes may be appropriate in this setting, following the guidelines for CVR use may have been a challenge for some, in particular those lacking a salary-based income or those being dissatisfied with $>3$ aspects of CVR use. Our sub-analysis of residual hormone levels in returned CVRs suggested increasing ring non-adherence over time. Among our behavioral sub-sample, no statistically significant factors for non-adherence either by self-report or by pharmacy record were found.

Clinical trials, observational studies, and medical practice have shown consistently high levels of satisfaction with NuvaRing ${ }^{\circledR} .20,21$ In general, women have indicated the ring is easy to use, effective, and convenient, with interference with sexual intercourse and local SEs (e.g., leukorrhea, vaginal discomfort, vaginitis) as the primary reasons cited for 
Table 2. Self-reported and pharmacy record non-adherence by sexually transmitted infections (STIs) and behavioral characteristics (n=185), Kisumu contraceptive vaginal ring study, Kisumu, Kenya, 2014-2015*

\begin{tabular}{|c|c|c|c|c|c|c|c|c|c|c|}
\hline \multirow[b]{2}{*}{ Predictor } & \multicolumn{4}{|c|}{$\begin{array}{c}\text { Non-adherence by self-report } \\
n=19 / 156(12.1 \%)\end{array}$} & \multirow[b]{2}{*}{$P$-value } & \multirow[b]{2}{*}{$\begin{array}{l}\text { Total, } \mathrm{n} \text { (col } \\
\% \text { ) }\end{array}$} & \multicolumn{3}{|c|}{$\begin{array}{l}\text { Non-adherence by pharmacy record } \dagger \\
n=106 / 185(57.3 \%)\end{array}$} & \multirow[b]{2}{*}{$P$-value } \\
\hline & $\begin{array}{l}\text { Total, } \mathrm{n} \\
\text { (col\%) }\end{array}$ & $\begin{array}{l}\text { Non- } \\
\text { adherent, } \mathrm{n} \\
\text { (\%) }\end{array}$ & $\begin{array}{l}\text { Prevalence } \\
\text { (\%) }\end{array}$ & $\begin{array}{l}\text { Prevalence } \\
\text { ratio }(95 \% \mathrm{Cl})\end{array}$ & & & $\begin{array}{l}\text { Non- } \\
\text { adherent, n } \\
\text { (col\%) }\end{array}$ & $\begin{array}{l}\text { Prevalence } \\
\text { (\%) }\end{array}$ & $\begin{array}{l}\text { Prevalence } \\
\text { ratio }(95 \% \mathrm{Cl})\end{array}$ & \\
\hline \multicolumn{4}{|c|}{ HIV incident infection: $\ddagger$} & N/A & N/A & & & & & \\
\hline Yes & $2(1.3)$ & $0(0.0)$ & 0.0 & & & $3(1.6)$ & $1(0.9)$ & 33.3 & $0.58(0.12,2.88)$ & 0.503 \\
\hline No & $154(98.7)$ & $19(100.0)$ & 12.3 & & & $182(98.4)$ & $105(99.1)$ & 57.7 & Ref. & \\
\hline \multicolumn{4}{|c|}{ Bacterial vaginosis incident infection:§ } & N/A & $\mathrm{N} / \mathrm{A}$ & & & & & \\
\hline Yes & $13(8.3)$ & $0(0.0)$ & 0.0 & & & $14(7.6)$ & $7(6.6)$ & 50.0 & $0.86(0.50,1.48)$ & 0.594 \\
\hline No & $143(91.7)$ & $19(100.0)$ & 13.3 & & & $171(92.4)$ & $99(93.4)$ & 57.9 & Ref. & \\
\hline \multicolumn{4}{|c|}{ Herpes simplex virus type-2 (HSV-2) incident infection:§ } & N/A & N/A & & & & & \\
\hline Yes & $4(2.6)$ & $0(0.0)$ & 0.0 & & & $5(2.7)$ & $2(1.9)$ & 40.0 & $0.69(0.23,2.04)$ & 0.505 \\
\hline No & $152(97.4)$ & $19(100.0)$ & 12.5 & & & $180(97.3)$ & $104(98.1)$ & 57.8 & Ref. & \\
\hline \multicolumn{11}{|c|}{ Chlamydia incident infection§ } \\
\hline Yes & $6(3.8)$ & $2(10.5)$ & 33.3 & $2.94(0.87,9.93)$ & 0.082 & $6(3.2)$ & $3(2.8)$ & 50.0 & $0.87(0.39,1.95)$ & 0.734 \\
\hline No & $150(96.2)$ & $17(89.5)$ & 11.3 & Ref. & & $179(96.8)$ & $103(97.2)$ & 57.5 & Ref. & \\
\hline \multicolumn{4}{|c|}{ Gonorrhea incident infection:§ } & N/A & $\mathrm{N} / \mathrm{A}$ & & & & N/A & N/A \\
\hline Yes & $1(0.6)$ & $0(0.0)$ & 0.0 & & & $2(1.1)$ & $2(1.9)$ & 100.0 & & \\
\hline No & $155(99.4)$ & $19(100.0)$ & 12.3 & & & $183(98.9)$ & $104(98.1)$ & 56.8 & & \\
\hline \multicolumn{4}{|c|}{ Syphilis incident infection:§ } & N/A & $\mathrm{N} / \mathrm{A}$ & & & & N/A & N/A \\
\hline Yes & $0(0.0)$ & $0(0.0)$ & 0.0 & & & $0(0.0)$ & $0(0.0)$ & 0.0 & & \\
\hline No & $156(100.0)$ & $19(100.0)$ & 12.2 & & & $185(100.0)$ & $106(100.0)$ & 57.3 & & \\
\hline \multicolumn{11}{|c|}{ Any STI or bacterial vaginosis incident infection:§ } \\
\hline Yes & $24(15.4)$ & $2(10.5)$ & 8.3 & $0.65(0.16,2.62)$ & 0.542 & $28(15.1)$ & $13(12.3)$ & 46.4 & $0.78(0.52,1.19)$ & 0.254 \\
\hline No & $132(84.6)$ & $17(89.5)$ & 12.9 & Ref. & & $157(84.9)$ & $93(87.7)$ & 59.2 & Ref. & \\
\hline \multicolumn{5}{|c|}{ Number of sexual partners in past 3 months: } & 0.555 & & & & & 0.775 \\
\hline No partners & $37(24.3)$ & $5(26.3)$ & 13.5 & $0.71(0.21,2.36)$ & 0.575 & $44(24.6)$ & $26(25.2)$ & 59.1 & $1.18(0.73,1.92)$ & 0.499 \\
\hline 1 partner & $94(61.8)$ & $10(52.6)$ & 10.6 & $0.56(0.19,1.61)$ & 0.281 & $113(63.1)$ & $66(64.1)$ & 58.4 & $1.17(0.75,1.82)$ & 0.495 \\
\hline 2 or more partners & $21(13.8)$ & $4(21.1)$ & 19.0 & Ref. & & $22(12.3)$ & $11(10.7)$ & 50.0 & Ref. & \\
\hline
\end{tabular}




\begin{tabular}{|c|c|c|c|c|c|c|c|c|c|c|}
\hline \multirow[b]{2}{*}{ Predictor } & \multicolumn{4}{|c|}{$\begin{array}{c}\text { Non-adherence by self-report } \\
\mathrm{n}=19 / 156(12.1 \%)\end{array}$} & \multirow[b]{2}{*}{$P$-value } & \multicolumn{5}{|c|}{$\begin{array}{c}\text { Non-adherence by pharmacy record } † \\
n=106 / 185(57.3 \%)\end{array}$} \\
\hline & $\begin{array}{l}\text { Total, n } \\
\text { (col\%) }\end{array}$ & $\begin{array}{c}\begin{array}{c}\text { Non- } \\
\text { adherent, } \mathrm{n} \\
(\%)\end{array} \\
\end{array}$ & $\begin{array}{l}\text { Prevalence } \\
(\%)\end{array}$ & $\begin{array}{l}\text { Prevalence } \\
\text { ratio }(95 \% \mathrm{Cl})\end{array}$ & & $\begin{array}{l}\text { Total, n (col } \\
\% \text { ) }\end{array}$ & $\begin{array}{c}\text { Non- } \\
\text { adherent, } \mathrm{n} \\
\text { (col\%) }\end{array}$ & $\begin{array}{l}\text { Prevalence } \\
(\%)\end{array}$ & $\begin{array}{l}\text { Prevalence } \\
\text { ratio }(95 \% \mathrm{Cl})\end{array}$ & $P$-value \\
\hline \multicolumn{11}{|c|}{ Vaginal sex in past 3 months: } \\
\hline Yes & $117(76.0)$ & $14(73.7)$ & 12.0 & $0.89(0.34,2.29)$ & 0.802 & $137(75.7)$ & $78(75.0)$ & 56.9 & $0.96(0.72,1.28)$ & 0.799 \\
\hline No & $37(24.0)$ & $5(26.3)$ & 13.5 & Ref. & & $44(24.3)$ & $26(25.0)$ & 59.1 & Ref. & \\
\hline \multicolumn{11}{|c|}{ Vaginal sex without a condom in past 3 months: } \\
\hline Yes & $112(72.7)$ & $12(63.2)$ & 10.7 & $0.64(0.27,1.52)$ & 0.315 & $130(71.8)$ & $72(69.2)$ & 55.4 & $0.88(0.68,1.15)$ & 0.350 \\
\hline No & $42(27.3)$ & $7(36.8)$ & 16.7 & Ref. & & $51(28.2)$ & $32(30.8)$ & 62.7 & Ref. & \\
\hline \multicolumn{11}{|c|}{ Anal sex in past 3 months: } \\
\hline Yes & $55(35.9)$ & $4(21.1)$ & 7.3 & $0.48(0.17,1.36)$ & 0.166 & $64(35.6)$ & $36(34.6)$ & 56.3 & $0.96(0.74,1.25)$ & 0.760 \\
\hline No & $98(64.1)$ & $15(78.9)$ & 15.3 & Ref. & & $116(64.4)$ & $68(65.4)$ & 58.6 & Ref. & \\
\hline \multicolumn{11}{|c|}{ Anal sex without a condom in past 3 months: } \\
\hline Yes & $52(34.2)$ & $3(15.8)$ & 5.8 & $0.36(0.11,1.18)$ & 0.092 & $59(33.1)$ & $33(32.0)$ & 55.9 & $0.95(0.72,1.25)$ & 0.716 \\
\hline No & $100(65.8)$ & $16(84.2)$ & 16.0 & Ref. & & $119(66.9)$ & $70(68.0)$ & 58.8 & Ref. & \\
\hline \multicolumn{11}{|c|}{ HIV(+/unknown) partners in past 3 months: } \\
\hline Yes & $50(33.6)$ & $5(26.3)$ & 10.0 & $0.71(0.27,1.85)$ & 0.481 & $57(32.4)$ & $28(27.2)$ & 49.1 & $0.78(0.58,1.05)$ & 0.101 \\
\hline No & $99(66.4)$ & $14(73.7)$ & 14.1 & Ref. & & $119(67.6)$ & $75(72.8)$ & 63.0 & Ref. & \\
\hline \multicolumn{4}{|c|}{ Unwanted/forced sex in past 3 months: } & N/A & N/A & & & & N/A & N/A \\
\hline Yes & $6(3.8)$ & $0(0.0)$ & 0.0 & & & $7(3.8)$ & $7(6.6)$ & 100.0 & & \\
\hline No & $150(96.2)$ & $19(100.0)$ & 12.7 & & & $178(96.2)$ & $99(93.4)$ & 55.6 & & \\
\hline \multicolumn{11}{|c|}{ Transactional sex or exchange sex in past 3 months: } \\
\hline Yes & $8(5.1)$ & $1(5.3)$ & 12.5 & $1.03(0.16,6.76)$ & 0.977 & $9(4.9)$ & $3(2.8)$ & 33.3 & $0.57(0.22,1.45)$ & 0.237 \\
\hline No & $148(94.9)$ & $18(94.7)$ & 12.2 & Ref. & & $176(95.1)$ & $103(97.2)$ & 58.5 & Ref. & \\
\hline
\end{tabular}

CI - confidence interval, STI - sexually transmitted infection

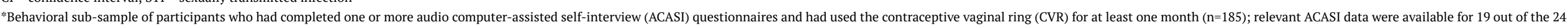
participants non-adherent by self-report and 106 out of the 110 participants non-adherent by pharmacy record; sample sizes fluctuate slightly for some variables due to missing data. Some percentages do not sum to 100 because of rounding.

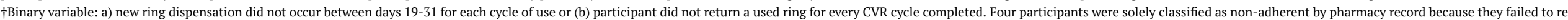
turn a used CVR for at least one follow-up visit.

$\Varangle$ Positive test at any time following CVR initiation.

\$Laboratory-confirmed diagnoses at study exit for women completing at least one 28-day CVR cycle. 


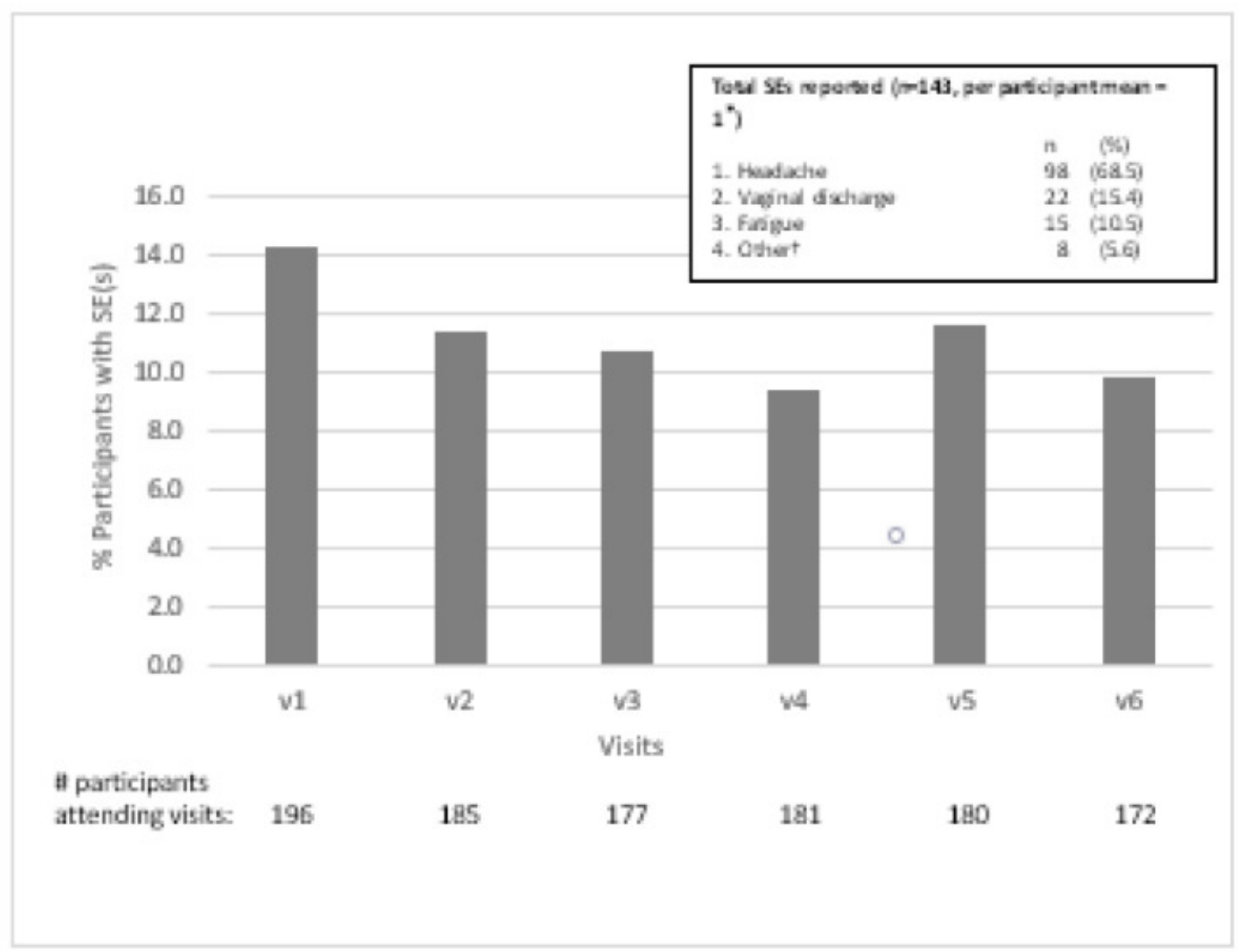

Figure 3. Proportion of participants $(n=202)$ reporting $\geqslant 1$ side effect $(S E)$ by Kisumu contraceptive vaginal ring study visit, Kisumu, Kenya, 2014-2015.

*Cumulative count of SEs reported by all participants over study follow-up; the per participant mean represents the average cumulative count of SEs reported by a participant over follow-up. $†$ Other category includes genital pain $(n=4)$, depression $(n=3)$, and elevated blood pressure $(n=1)$.

either discontinuing or disliking the ring. ${ }^{21,22}$ Common reasons for disliking the CVR were similar for women in our trial. Presumably, with increased consumer education, as well as an improved understanding of user preferences, these barriers can be overcome.

Non-adherence ranging from $9 \%$ to $20 \%$ by self-report have been found in other NuvaRing ${ }^{\circledR}$ studies $^{21,23}$ and was slightly lower (8\%) in a recent dapivirine ring HIV prevention trial conducted in multiple African countries. ${ }^{24}$ Our bypharmacy-record measure may have under-estimated nonadherence. Other studies have defined NuvaRing ${ }^{\circledR}$ cycle non-adherence as a ring cycle that (a) extended 48 hours beyond day 22, and (b) the length of ring-free period was lengthened by more than 24 hours from day $8 .^{25}$ Notably, these studies collected diaries and tracking logs completed by the participant as opposed to our method, which involved calculations based on pharmacy dates for dispensation of new CVRs and return dates of a used CVRs. As suggested by others, non-adherence for longer-acting ARV rings require cumulative measurement over time that could benefit from non-invasive electronic and biometric monitoring technologies requiring minimal participant effort. ${ }^{26}$

Other studies have found decreased non-adherence among women with an independent income. ${ }^{4}$ Women with their own source of income may have greater family planning decision-making input than women who depend on their partners, or women who have variable income (eg, seasonal, casual, or temporary work). While additional research is needed, economic empowerment interventions suggest that most programs show reproductive health improvements. ${ }^{27}$

A recent systematic review of contraceptive use in subSaharan Africa suggests that misinformation and concerns about perceived SEs are common barriers in accepting and using modern contraceptive methods. ${ }^{28}$ As measured in our study, we did not find that SE concerns and tolerability were barriers to CVR use.

A number of limitations are associated with this study. The generalizability of our findings is limited due to our study design and sampling approach. While women in our sample were able to use a novel intravaginal ring to prevent pregnancy, which may have implications for future use of rings to prevent HIV and other STIs in this high-burden region, caution is warranted with such an interpretation.

A gold standard for assessing adherence to modern contraceptive methods is not available. ${ }^{29}$ Our definitions of particular measures, in particular non-adherence by selfreport, non-adherence by pharmacy record, may have pro- 
duced imprecisions with either observation or measurement process. Measures for assessing CVR non-adherence in the literature are largely limited to self-report and subject to under-reporting of non-adherent behavior. We acknowledge that some women in our study could have had on-time CVR dispensation (between days 19 and 31 for each monthly cycle) and returned a used ring; yet, they could have been partially or completely non-adherent. Similar to self-report, a by-pharmacy-record measure is suboptimal in reliably assessing non-adherence. HIV risk perceptions along with HIV stigma, which were not measured in our study, may have created reticence to initiate ring use and influenced non-adherence behavior. Self-reported non-adherence and behavioral data may be subject to recall or social desirability biases. Performing residual hormone analysis on the returned rings from all women in our sample was not feasible. Moreover, such objective measures may not be entirely clear-cut in their ability to distinguish inconsistent users from consistent users. ${ }^{29}$ Given small count, our pregnancy rate was estimated with low precision and reliability, and should be interpreted with caution. Lastly, NuvaRing ${ }^{\circledR}$ may offer a slight allowance for imperfect use given that removal of the ring for up to three hours for sexual or hygiene purposes does not compromise contraceptive efficacy.

\section{CONCLUSIONS}

While vaginal rings may be a highly effective but underutilized contraceptive approach and potentially a preferred future HIV prevention method for some women, perfect use will be a challenge. Apart from measurement challenges of non-adherence in clinical trial settings, real world non-adherence must be carefully accounted for in development of any multipurpose vaginal ring product. Economic empowerment interventions, in particular those that emphasize consistent and partner-independent income options, may mitigate non-adherence to modern contraceptive methods such as a CVR. To better understand non-adherent behavior, it may be necessary to go beyond looking for demographic and behavioral factors to explain CVR non-adherence, such as analyzing participants' relationship with power dynamics, self-assertion, and sexuality. Lastly, to further minimize non-adherence, preemptively addressing CVR dissatisfaction and expanding education on CVR features may be beneficial.

\section{ACKNOWLEDGEMENTS}

The authors would like to thank the study participants for their time and contribution to this study as well as the broader Kisumu community for its support. The authors would also like to thank Beatrice Nyagol, Kenneth Ondeng'e, Lawrence Opado, Richard Ndivo, and members of the study implementation team. The authors extend a special thanks to Siobhan O'Connor, Clyde Hart, and David Schnabel for their technical guidance and support.

\section{DISCLAIMER}

The findings and conclusions in this report are those of the authors and do not necessarily represent the views of the United States Centers for Disease Control and Prevention (CDC) or the Department of Health and Human Services. The Kenya Medical Research Institute (KEMRI) Director and the KEMRI Publication Review Committee gave permission to publish this manuscript.

\section{FUNDING}

The Centers for Disease Control and Prevention supported this work under PN1757, 2014-2015.

\section{CLINICAL TRIAL INFORMATION}

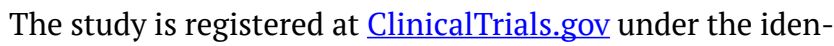
tifier NCT02529683.

\section{COMPETING INTERESTS}

The authors have completed the Unified Competing Interest form at http://www.icmje.org/coi_disclosure.pdf (available on request from the corresponding author) and declare no conflict of interest.

\section{CORRESPONDENCE TO:}

Eleanor McLellan-Lemal

Centers for Disease Control and Prevention

1600 Clifton Rd., MS-E45

Atlanta, GA 30329

United States of America

egm4@cdc.gov 


\section{REFERENCES}

1. Sitruk-Ware R, Nath A, Mishell DR Jr.

Contraception technology: past, present and future.

Contraception. 2013;87(3):319-330. doi:10.1016/j.cont raception.2012.08.002

2. Abdool Karim Q, Abdool Karim SS, Frohlich JA, et al. Effectiveness and safety of tenofovir gel, an antiretroviral microbicide, for the prevention of HIV infection in women. Science.

2010;329(5996):1168-1174. doi:10.1126/science.1193 $\underline{748}$

3. Bahamondes L, Bahamondes MV. New and emerging contraceptives: a state-of-the-art review. Int J Womens Health. 2014;6:221-234. doi:10.2147/ijw h.s46811

4. Marrazzo JM, Ramjee G, Richardson BA, et al. Tenofovir-based preexposure prophylaxis for HIV infection among African women. $N$ Engl J Med. 2015;372(6):509-518. doi:10.1056/neimoa1402269

5. Thurman A, Clark M, Hurlburt J, Doncel G. Intravaginal rings as delivery systems for microbicides and multipurpose prevention technologies. Int J Womens Health. 2013;5:695-708. d oi:10.2147/ijwh.s34030

6. Guttmacher Institute. Contraceptive use in the United States fact sheet. Published 2016. Accessed April 30, 2018. https://www.guttmacher.org/fact-shee t/contraceptive-use-united-states

7. Oddsson K, Leifels-Fischer B, de Melo NR, et al. Efficacy and safety of a contraceptive vaginal ring (NuvaRing) compared with a combined oral contraceptive: a 1-year randomized trial. Contraception. 2005;71(3):176-182. doi:10.1016/j.cont raception.2004.09.001

8. Stewart FH, Brown BA, Raine TR, Weitz TA, Harper CC. Adolescent and young women's experience with the vaginal ring and oral contraceptive pills. J Pediatr Adolesc Gynecol. 2007;20(6):345-351. doi:10.1016/i.jp ag.2007.06.001

9. van der Straten A, Montgomery ET, Cheng H, et al. High acceptability of a vaginal ring intended as a microbicide delivery method for HIV prevention in African women. AIDS Behav. 2012;16(7):1775-1786. $\underline{\mathrm{d}}$ oi:10.1007/s10461-012-0215-0

10. Brache V, Payán LJ, Faundes A. Current status of contraceptive vaginal rings. Contraception. 2013;87(3):264-272. doi:10.1016/j.contraception.201 2.08.037
11. Ahrendt HJ, Nisand I, Bastianelli C, et al. Efficacy, acceptability and tolerability of the combined contraceptive ring, NuvaRing, compared with an oral contraceptive containing $30 \mu \mathrm{g}$ of ethinyl estradiol and $3 \mathrm{mg}$ of drospirenone. Contraception. 2006;74(6):451-457. doi:10.1016/i.contraception.200 $\underline{6.07 .004}$

12. Woodsong C, MacQueen K, Amico KR, et al. Microbicide clinical trial adherence: insights for introduction. J Int AIDS Soc. 2013;16:18505. doi:10.74 48/ias.16.1.18505

13. Tolley EE, Severy LJ. Integrating behavioral and social science research into microbicide clinical trials: challenges and opportunities. Am J Public Health. 2006;96:79-83. doi:10.2105/ajph.2004.043471

14. World Bank. Promoting women's agency. In: World Development Report 2012: Gender Equality and Development. World Bank; 2012.

15. Novák A, de la Loge C, Abetz L. Development and validation of an acceptability and satisfaction questionnaire for a contraceptive vaginal ring, NuvaRing. Pharmacoeconomics. 2004;22(4):245-256. ㅁ oi:10.2165/00019053-200422040-00003

16. Wieder DR, Pattimakiel L. Examining the efficacy, safety, and patient acceptability of the combined contraceptive vaginal ring (NuvaRing). Int J Womens Health. 2010;2:401-409. doi:10.2147/ijwh.s6162

17. McNutt LA, Wu C, Xue X, Hafner JP. Estimating the relative risk in cohort studies and clinical trials of common outcomes. Am J Epidemiol. 2003;157(10):940-943. doi:10.1093/aje/kwg074

18. Hilbe JM, Hardin J, Hardin H. Generalized Estimating Equations. 2nd ed. CRC Press; 2003.

19. Cohen J. A coefficient of agreement for nominal scales. Educ Psychol Meas. 1960;20:37-46. doi:10.117 7/001316446002000104

20. Roumen FJ, op ten Berg MM, Hoomans EH. The combined contraceptive vaginal ring (NuvaRing ${ }^{\circledR}$ ): first experience in daily clinical practice in the Netherlands. Eur J Contracept Reprod Health Care. 2006;11:14-22. doi:10.1080/13625180500389547

21. Roumen FJ, Mishell DR Jr. The contraceptive vaginal ring, NuvaRing ${ }^{\circledR}$, a decade after its introduction. Eur J Contracept Reprod Health Care. 2012;17(6):415-427. doi:10.3109/13625187.2012.7135 $\underline{35}$ 
22. Roumen FJ. Review of the combined contraceptive vaginal ring, NuvaRing ${ }^{\circledR}$. Ther Clin Risk Manag. 2008;4:441-451. doi:10.2147/tcrm.s1964

23. Dieben TO, Roumen FJ, Apter D. Efficacy, cycle control, and user acceptability of a novel combined contraceptive vaginal ring. Obstet Gynecol. 2002;100:585-593.

24. Nel A, Bekker LG, Bukusi E, et al. Safety, acceptability and adherence of dapivirine vaginal ring in a microbicide clinical trial conducted in multiple countries in sub-Saharan Africa. PLoS ONE. 2016;11(3):e0147743. doi:10.1371/journal.pone.0147 $\underline{743}$

25. Bruni V, Pontello V, Luisi S, Petraglia F. An openlabel, multicentre trial to evaluate the vaginal bleeding pattern of the combined contraceptive vaginal ring NuvaRing ${ }^{\circledR}$. Eur J Obstet Gynecol Reprod Biol. 2008;139:65-71. doi:10.1016/j.ejogrb.2008.02.00 $\underline{1}$
26. MacQueen KM, Tolley EE, Owen DH, et al. An interdisciplinary framework for measuring and supporting adherence in HIV prevention trials of ARV-based vaginal rings. J Int AIDS Soc. 2014;17(3(Suppl 2)):19158. doi:10.7448/ias.17.3.1915 $\underline{8}$

27. Reed E, West BS, Salazar M, Monroy RV. Economic empowerment to improve sexual and reproductive health among women and girls. In: Global Perspectives on Women's Sexual and Reproductive Health across the Lifecourse. Springer International Publishing; 2018:297-315. doi:10.1007/978-3-319-60417-6 17

28. Blackstone SR, Nwaozuru U, Iwelunmor J. Factors influencing contraceptive use in sub-Saharan Africa: a systematic review. Int $Q$ Community Health Educ. 2017;37(2):79-91. doi:10.1177/0272684x16685254

29. Smith C, Edwards P, Free C. Assessing the validity and reliability of self-report data on contraception use in the Mobile Technology for Improved Family Planning (MOTIF) randomised controlled trial. Reprod Health. 2018;15:50. doi:10.1186/s12978-018-0494-7 